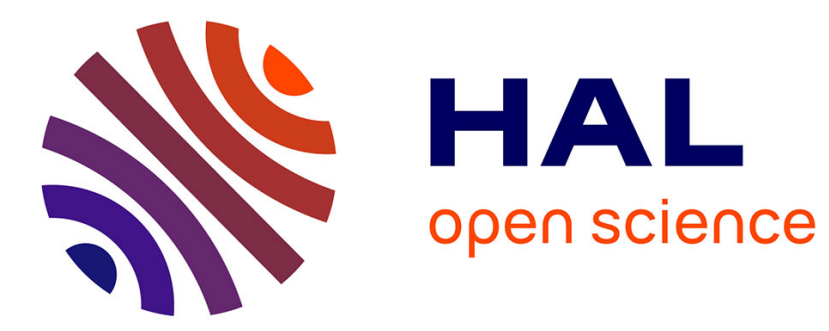

\title{
On the Discrete Lot-Sizing and Scheduling Problem with Sequence-Dependent Changeover Time
}

Céline Gicquel, Michel Minoux, Yves Dallery

\section{To cite this version:}

Céline Gicquel, Michel Minoux, Yves Dallery. On the Discrete Lot-Sizing and Scheduling Problem with Sequence-Dependent Changeover Time. Operations Research Letters, 2009, 37 (1), pp.32-36. 10.1016/j.orl.2008.10.001 . hal-01170355

\section{HAL Id: hal-01170355 \\ https://hal.science/hal-01170355}

Submitted on 3 Feb 2016

HAL is a multi-disciplinary open access archive for the deposit and dissemination of scientific research documents, whether they are published or not. The documents may come from teaching and research institutions in France or abroad, or from public or private research centers.
L'archive ouverte pluridisciplinaire HAL, est destinée au dépôt et à la diffusion de documents scientifiques de niveau recherche, publiés ou non, émanant des établissements d'enseignement et de recherche français ou étrangers, des laboratoires publics ou privés. 


\title{
On the discrete lot-sizing and scheduling problem with sequence-dependent changeover times
}

\author{
C. Gicquel ${ }^{a, 1}$, M. Minoux ${ }^{b}$, Y. Dallery ${ }^{a}$ \\ ${ }^{a}$ Ecole Centrale Paris, Laboratoire de Génie Industriel \\ Grande Voie des Vignes, 92290 Chatenay-Malabry, France \\ ${ }^{b}$ Laboratoire d'Informatique de Paris 6 \\ 4 place Jussieu, 75005 Paris, France
}

\begin{abstract}
We consider the discrete lot-sizing and scheduling problem with sequence-dependent changeover costs and times and propose to solve it as a mixed-integer program using a commercial solver. Our approach is based on the extension of an existing tight formulation for the case without changeover times. Computational results confirm the benefits of the proposed solution procedure.
\end{abstract}

Keywords: Lot-sizing, Sequence-dependent setup costs, Setup times, Mixed-integer programming

\section{Introduction}

A wide variety of models for production planning and inventory management has been investigated in operations research. Among them, capacitated lotsizing models aim at determining the optimal timing and level of production complying with given capacity restrictions and such that demand for all products is satisfied without backlogging. Recent overviews on the lot-sizing literature can be found among others in [2] and [4].

In the present paper, the discrete lot-sizing and scheduling problem (DLSP) is considered. The DLSP relies on several basic assumptions (see e.g. [3]) :

- Demand for products is deterministic and timevarying.

- The production plan is established for a finite time horizon subdivided in several discrete periods.

- At most one item can be produced per period ("small bucket" model) and the facility processes either one product at full capacity or is completely idle ("all-or-nothing assumption").

- Costs to be minimized are the inventory holding costs and the changeover costs.

Here the single level single machine variant of this problem is studied: all items to be produced are end items and share the same constrained resource. In the DLSP, it is assumed that there is a changeover between two production runs for different items, resulting in a changeover cost and/or a changeover time. Changeover costs and times can depend either on the next item only (sequenceindependent case) or on the sequence of items (sequence-dependent case). Significant changeover

${ }^{1}$ Corresponding author

Tel.: +33 1411318 04; fax: +33141 131272 .

E-mail address: celine.gicquel@ecp.fr times which consume scarce production capacity tend to further complicate the problem. We consider here the most difficult variant: the DLSP with sequence-dependent changeover costs and times (denoted DLSPSD in the sequel).

The DLSP has received much attention in the literature. Complexity results for this problem can be found in [8]. They show that the single machine multi-product case without setup times is NP-hard and that the problem of finding a feasible solution in the presence of sequence-independent setup times is NP-complete. We deal here with the extension of this problem to the case of sequencedependent changeover costs and times. The DLSPSD under study in the present paper is thus NPhard.

We now discuss into more detail specific contributions on the DLSPSD. [9] reformulate the problem as a travelling salesman problem with time windows and use a dynamic programming-based algorithm to solve it. [5] show the equivalence between the DLSPSD and the batch sequencing problem (BSP) and use a specific branch and bound type algorithm for solving the BSP to optimality. In both papers, the mixed-integer programming formulation proposed for the problem is weak and does not provide lower bounds good enough to solve the problem using a commercial solver (see results in section 3.2). However, as pointed out by [7], there is now a good knowledge about the "right" way to formulate many simple production planning submodels as mixed-integer programs and, thanks to it, many practical production planning problems can be (approximately) solved using commercial solvers. To the best of our knowledge, these results 
have not yet been exploited to solve the DLSPSD. In the present paper, we attempt to close this gap by proposing a new tight formulation for this specific variant of the problem.

The purpose of this paper is thus to introduce a strengthened formulation for the DLSP with sequence-dependent changeover costs and times. This formulation is an extension of the formulation proposed by [11] for the DLSP with sequencedependent changeover costs and zero changeover times. Thanks to this strengthened formulation, the lower bounds provided by the linear relaxation of the problem are significantly better, enabling a branch and bound type procedure to solve the problem more efficiently.

The paper is organized as follows. In section 2 , we present the proposed tight formulation for the DLSPSD. In section 3, we discuss the results of some computational experiments carried out to evaluate it.

\section{A tight formulation for the DLSPSD}

We present a tight formulation for the DLSP with sequence-dependent changeover costs and times. This formulation is an extension of the formulation proposed by [11] for the DLSP with sequencedependent changeover costs and zero changeover times. In the sequel, we denote it the DSLPSD2 formulation whereas we denote DLSPSD1 the formulation proposed by [9].

\subsection{Basic formulation}

We wish to optimize the production schedule for a set of $N$ items over an horizon featuring $T$ planning periods. A period is indexed by $t=1, \ldots, T$, an item by $i=0, . ., N$. We agree to use item $i=0$ to represent idle periods.

We use the following notation:

- $d_{i t}$ : demand (in units) for item $i$ in period $t$.

- $P_{i t}$ : production capacity (in units per period) for item $i$ in period $t$.

- $h_{i}$ : holding costs per unit and period for item $i$.

- $c_{i j}$ : changeover costs from item $i$ to item $j$.

- $T_{i j}$ : changeover time from item $i$ to item $j . T_{i j}$ is assumed to be an integer number of planning periods.

Decision variables are defined as follows:

- $I_{i t}$ : inventory level corresponding to item $i$ at the end of period $t$.

- $y_{i t}$ : setup variables. $y_{i t}$ equals 1 if the resource is setup for production of item $i$ in period $t$, and 0 otherwise.

$-w_{i j t}$ : changeover cost variables. If $T_{i j}>0, w_{i j t}$ equals 1 during the first period of a changeover from item $i$ to item $j$, and 0 otherwise. If $T_{i j}=0$, $w_{i j t}$ equals 1 in the first period of production of $j$, and 0 otherwise.

$-v_{t}$ : changeover time variables. $v_{t}$ equals 1 during each period in which a changeover between two items occurs, and 0 otherwise.

In the mixed-integer formulation proposed in table 1 , the objective (3) minimizes the sum of inventory holding costs and changeover costs. Note that, in the DLSPSD2 formulation, variables $w_{i i t}$ are introduced: $w_{i i t}=1$ means that the resource is setup for item $i$ both in period $t-1$ and in period $t$, i.e. that a production run for item $i$ takes place over periods $t-1$ and $t$.

Constraints (4) express the inventory balance. Together with constraints (8), they ensure that demand for each item is fulfilled without backlogging.

Equalities (5) and (6) link the setup variables with the changeover cost variables. (5) guarantee that item $i$ can be produced in period $t-1$ if and only if a changeover from $i$ to another item $j$ (possibly $j=i$ ) takes place at the beginning of period $t$. Similarly, (6) guarantee that item $j$ can be produced in period $t$ if and only if a changeover from another item $i$ (possibly $i=j$ ) to item $j$ begins early enough (i.e. in period $t-T_{i j}$ ) to be finished at the beginning of period $t$.

(7) ensure that in each period, the resource either produces a single product at full capacity, or is idle (i.e $y_{0 t}=1$ ), or is in transition between two items (i.e. $v_{t}=1$ ).

The binary character of the setup variables is represented by (9). (10) and (11) state the nonnegativity of the changeover variables: observe, as pointed out by [1], that thanks to constraints (5)(7) and (9), there is no need to define variables $w_{i j t}$ and $v_{t}$ as binary variables.

\section{$2.2 \quad$ Valid inequalities}

As shown in [11] for the case without changeover times, the DLSPSD2 formulation can be further strengthened through a family of valid inequalities adapted from the ones developed by [10]. We investigate here an extension of this idea to the case of positive changeover times and propose a family of valid inequalities for the problem (3)-(11).

This can be done using the assumption of Wagner-Whitin costs, constant capacity and no backlogging. In this case, demands and production capacity can be normalized without loss of generality: $d_{i t} \in\{0,1\}$ and $P_{i t}=1$. We first introduce some additional notation:

- $D_{i, t, \tau}$ : cumulated demand for item $i$ in the interval $\{t, \ldots, \tau\}$. Demand on item $i$ is binary so that $D_{i, t, \tau}$ is equal to the number of positive demand 
periods for $i$ in $\{t, \ldots, \tau\}$.

- $S_{i, q}: q^{t h}$ positive demand period for item $i$. Note that $S_{i, D_{i, 1, t}+q}$ denotes the $q^{\text {th }}$ period with positive demand for item $i$ after period $t$.

We also introduce the start-up variables $z_{i t}$ defined as follows: $z_{i t}$ equals 1 if the production of a new lot of item $i$ starts at the beginning of period $t, 0$ otherwise. These start-up variables are linked to the changeover variables by the equations:

$$
z_{j t}=\sum_{i: i \neq j} w_{i j, t-T_{i j}} \quad \forall j=1 \ldots N, \forall t=1 \ldots T
$$

Equalities (1) state that the production of a new lot of item $j$ begins in period $t$ if and only if a changeover from another item $i \neq j$ starts "early enough" (i.e. in period $t-T_{i j}$ ) to be finished at the beginning of period $t$.

With this notation, we have:

Proposition 1. The following inequations (2) are valid inequalities for the DLSP with sequencedependent changeover costs and times:

$$
\begin{aligned}
& I_{i t} \geq \sum_{q=1}^{p}\left(1-y_{i, t+q}-\sum_{\tau=t+q+1}^{S_{i, D_{i, 1, t}+q}} z_{i \tau}\right) \\
& \forall i=1 \ldots N, \forall t=1 \ldots T, \forall p \in\left\{0 \ldots D_{i, t+1, T}\right\}
\end{aligned}
$$

Proof. A sketch of proof is as follows. First note that $y_{i, t+q}+\sum_{\tau=t+q+1}^{S_{i, D_{i, 1, t}+q}} z_{i \tau}=0$ if and only if the resource is not setup for item $i$ in period $t+q$ and no startup for $i$ takes place between the period $t+q+1$ and the period where the $q^{t h}$ demand after period $t$ occurs, i.e. if and only if no production of item $i$ is possible in the interval $\left\{t+q, \ldots, S_{i, D_{i, 1, t}+q}\right\}$. In this case, the quantity needed to satisfy the $q^{\text {th }}$ demand after period $t$ should be in stock at the end of period $t$. Thus we see that constraints (2) force an increase of the stock of item $i$ at the end of period $t$ by one for each index $q$ for which no production occurs in the interval $\left\{t+q, \ldots, S_{i, D_{i, 1, t}+q}\right\}$. A detailed proof of the validity of (2) can easily be derived from the above (see also [10]).

In the computational experiments to be presented in section 3 , we use a standard cutting-plane generation method (see e.g. [6]) to strengthen the DSLPSD2 formulation by adding violated valid inequalities (2). The resulting improved formulation is denoted DLSPSD2*.

\subsection{Remark}

It is interesting to keep in mind that equalities (5)(6) can be interpreted as flow conservation constraints in a network.

More precisely, we consider a graph $\mathcal{G}=(\mathcal{V}, \mathcal{A})$. A node $v \in \mathcal{V}$ corresponds to a item-period pair $(i, t)$. There is an oriented arc $a \in \mathcal{A}$ from node $v_{1}$ to node $v_{2}$ if and only if $v_{1}=(i, t)$ and $v_{2}=$ $\left(j, t+T_{i j}+1\right)$. The setup variable $y_{i t}$ corresponds to the flow through node $(i, t)$ and the changeover variable $w_{i j, t+1}$ corresponds to the flow between node $(i, t)$ and node $\left(j, t+T_{i j}+1\right)$. With this interpretation, a production sequence on the resource corresponds to a flow of a single unit through the network, starting from a node $(i, 0)$ and arriving in a node $(j, T)$. Thus equalities (5) and (6) can be seen as flow conservation constraints, stating that the flow through a node is equal to the sum of the flows on the arcs directed away from it or to the sum of the flows on the arcs directed toward it.

Due to the presence of positive changeover times, the structure of the graph $\mathcal{G}$ used here is seen to be different from the one used in [1]. Namely, when there are no changeover times, all arcs in graph $\mathcal{G}$ link pairs of nodes related to successive planning periods, which is not the case anymore with positive changeover times.

\section{Computational results}

In this section, we discuss the results of some computational experiments carried out to evaluate the DLSPSD2* formulation proposed in section 2 .

\subsection{Problem instance generation}

We created several sets of randomly generated instances following the procedure described in [9] and [5]. The reader is referred to these references for more details. The generated instances differ with respect to the following characteristics:

- Problem dimension: The problem dimension is represented by the number of products $N$ and the number of periods $T$. We use 7 different item-period combinations, namely $(N, T)=\{(5,20),(10,40)$, $(5,60),(10,60),(15,60),(10,90),(15,90)\}$, leading to 7 instance sets denoted sets A to G.

- Production capacity utilization: Production capacity utilization $\rho$ is defined as the ratio between the total cumulated demand and the total cumulated available capacity. Because changeover times are nonzero, we experimented different medium values for $\rho$ : $\rho$ was varied between 0.5 and 0.75 , in steps of 0.05 .

For each possible combination of problem dimension and production capacity utilization, 5 problems were generated, resulting in $7 \times 6 \times 5=210$ instances. All tests were run on a Pentium 4 $(2.8 \mathrm{Ghz})$ with $505 \mathrm{Mb}$ of RAM, running under Windows XP. We used a standard MIP software (CPLEX 8.1.0) with the solver default settings to solve the problem, using either the DLSPSD1 formulation presented in [9] or the DSLPSD2 and DLSPSD2* formulations presented in section 2 . 


\subsection{Comparison of DSLPSD1, DL- SPSD2 and DLSPSD2* formula- tions}

We first carried out some computational experiments in order to evaluate the reformulation proposed in section 2.1 and the family of valid inequalities derived in section 2.2. The comparison has been limited to the smallest instances (sets A and B) since computation time limits are exceeded for set B instances with the DLSPSD1 formulation.

Table 2 shows the results obtained with the DSLPSD1, DLSPSD2 and DLSPSD2* formulations for the sets A and B instances. For each formulation, we provide:

- Variables and Constraints: the average number of variables and constraints.

- \#VI: for the DLSPSD2* formulation, the average number of valid inequalities of type (2) added by the cutting-plane generation procedure.

- \#Opt: the number of instances out of the corresponding 30 instances that could be solved to optimality within 20 minutes of computation.

- $G a p_{0}$ : the integrality gap, i.e. the relative difference between the lower bound provided by the linear relaxation of the problem and the value of an optimal solution. For the DLSPSD2* formulation, we consider the lower bound obtained after the cutting-plane generation procedure has stopped.

- \#Nodes: the number of nodes of the search tree explored before a guaranteed optimal solution is found or the computation time limit of 20 minutes is reached.

- $C P U_{I P}$ : the computation time in seconds required to find a guaranteed optimal solution. If one could not be found, we use the computation time limit of 1200 seconds.

- Gap: the gap obtained after 20 minutes of computation between the best integer solution and the best lower bound found.

For performance measures $G_{a p}$, Nodes, $C P U_{I P}$ and $G a p$, we provide the average value (on the first line) and the minimum and maximum values (in brackets on the second line) for the considered set of randomly generated instances.

Table 2 shows that the results obtained with the DLSPSD2* formulation are much better than the ones obtained with the DLSPSD1 formulation. Namely, computation times are significantly reduced and more instances can be solved to optimality within the time limit while using the DLSPSD2* formulation.

This can be explained by the combination of two advantages:

1. The lower bounds provided by the linear relaxation of the DLSPSD2* formulation are much better than the ones obtained with the DLSPSD1 formulation. This formulation improvement is achieved to a large extent thanks to the use of a small number of valid inequalities (2). This can be seen e.g. for set A instances for which the integrality gap is reduced in average from $84 \%$ with the DLSPSD1 formulation to $59 \%$ with the basic DLSPSD2 formulation and $4 \%$ with the strengthened DLSPSD2* formulation.

2. The MIP size (number of variables and constraints) is significantly reduced with the DLSPSD2* formulation. As a consequence, the time spent at each node of the branch and bound tree to solve the linear relaxation is shorter. This size reduction is explained by the fact that using the DLSPSD2* formulation, there is no need to introduce explicit changeover time variables $v_{i j t}$ for each possible transition between pairs of items $(i, j)$. Moreover, the set of equalities (5)-(6) are sufficient to ensure that positive changeover times between production runs for different items are respected. As a consequence, the numerous inequalities needed in the DLSPSD1 formulation to link changeover time variables to setup and changeover cost variables can be eliminated from the formulation.

Thus, thanks to tighter lower bounds and a reduced MIP size, the efficiency of the branch and bound procedure embedded in CPLEX solver is significantly improved while using the DLSPSD $2 *$ formulation.

\subsection{Results with the MIP formula- tion DLSPSD2*}

In order to further validate our approach, we carried out additional computational experiments. More precisely, we considered instances similar to the ones studied in [5] and [9], i.e. instances for which $(N, T)=\{(5,60),(10,60)\}$ (sets C-D). We also used 3 additional sets of larger instances for which $(N, T)=\{(15,60),(10,90),(15,90)\}$ (sets EG).

Table 3 displays the detailed results obtained with the DLSPSD2* formulation. We observe that: - For medium size instances (sets C-D), $98 \%$ of the generated instances could be solved to optimality within 20 minutes of computation.

- For large size instances (sets E-G), $42 \%$ of the generated instances could be solved to optimality within the computation time limits. Moreover the average remaining gap obtained after 20 minutes of computation between the best integer solution and the best lower bound found is small $(3.6 \%$ on average).

Thus, even if the proposed approach was implemented on a computer with more computing power, these results suggest the potential of the MIP modelling approach to solve instances larger than the 
ones considered in [5] and [9].

\section{References}

[1] G. Belvaux, L.A. Wolsey, Modelling practical lot-sizing problems as mixed-integer programs, Management Science 47(7) (2001) 993-1007.

[2] A. Drexl, A. Kimms, Lot sizing and scheduling - Survey and extensions, European Journal of Operational Research 9 (1997) 221-235.

[3] B. Fleischmann, The discrete lot sizing and scheduling problem, European Journal of Operational Research 44 (1990) 337-348.

[4] R. Jans, Z. Degraeve, Meta-heuristics for dynamic lot sizing: a review and comparison of solution approaches, European Journal of Operational Research 177 (2007) 1855-1875.

[5] C. Jordan, A. Drexl, Discrete lotsizing and scheduling by batch sequencing, Management Science 44(5) (1998) 698-713.

[6] G. Nemhauser, L.A. Wolsey, Integer and Combinatorial Optimization., John Wiley \& Sons, 1988.
[7] Y. Pochet, L.A. Wolsey, Production planning by mixed integer programming, Springer Science, 2006.

[8] M. Salomon, L.G. Kroon, R. Kuik R, L.N. van Wassenhove, Some extensions of the discrete lotsizing and scheduling problem, Management Science 37(7) (1991) 801-812.

[9] M. Salomon, M. Solomon, L.N. van Wassenhove, Y. Dumas, S. Dauzère-Pérès, Solving the discrete lotsizing and scheduling problem with sequence dependent set-up costs and setup times using the travelling salesman problem with time windows, European Journal of Operational Research 100 (1997) 494-513.

[10] C.A. van Eijl, C.P.M. van Hoesel, On the discrete lot-sizing and scheduling problem with Wagner-Whitin costs, Operations Research Letters 20 (1997) 7-13.

[11] L.A. Wolsey, Solving multi-item lot-sizing problems with an MIP solver using classification and reformulation, Management Science 48(12) (2002) 1587-1602. 


$$
\begin{array}{ll}
\min \sum_{i=1}^{N} \sum_{t=1}^{T} h_{i} I_{i t}+\sum_{i=0}^{N} \sum_{j=0}^{N} \sum_{t=1}^{T} c_{i j} w_{i j t} & \\
I_{i t}=I_{i, t-1}+P_{i t} y_{i t}-d_{i t} & \forall i=1 \ldots N, \forall t=1 \ldots T \\
y_{i, t-1}=\sum_{j=0}^{N} w_{i j t} & \forall i=0 \ldots N, \forall t=1 \ldots T \\
y_{j t}=\sum_{i=0 \ldots N \text { st } t-T_{i j}>0} w_{i j, t-T_{i j}} & \forall j=0 \ldots N, \forall t=1 \ldots T \\
\sum_{i=0}^{N} y_{i t}+v_{t}=1 & \forall t=0 \ldots T \\
I_{i t} \geq 0 & \forall i=1 \ldots N, \forall t=1 \ldots T \\
y_{i t} \in\{0,1\} & \forall i=0 \ldots N, \forall t=0 \ldots T \\
w_{i j t} \in[0,1] & \forall i=0 \ldots N, \forall j=0 \ldots N, \forall t=1 \ldots T \\
v_{t} \in[0,1] & \forall t=0 \ldots T
\end{array}
$$

Table 1: Mixed integer formulation DLSPSD2 
set $\mathrm{A}$ set $\mathrm{B}$

\begin{tabular}{lcccccc} 
MIP formulation & DLSPSD1 & DLSPSD2 & DLSPSD2 & DLSPSD1 & DLSPSD2 & DLSPSD2* \\
\hline Variables & 1167 & 960 & 960 & 7880 & 5720 & 5720 \\
Constraints & 2392 & 361 & 361 & 19946 & 1321 & 1321 \\
\#VI & & - & 124 & - & - & 479 \\
\hline \#Opt & 27 & 30 & 30 & 0 & 0 & 30 \\
\hline Gap $(\%)$ & 84 & 59 & 4 & 92 & 68 & 5 \\
& {$[65 ; 95]$} & {$[47 ; 69]$} & {$[0 ; 12]$} & {$[80 ; 96]$} & {$[57 ; 73]$} & {$[0 ; 13]$} \\
\#Nodes & 49362 & 738 & 3 & 2497 & 35497 & 100 \\
& {$[16 ; 191523]$} & {$[11 ; 2780]$} & {$[0 ; 19]$} & {$[391 ; 4969]$} & {$[24705 ; 52968]$} & {$[2 ; 319]$} \\
CPU $_{\text {IP }}(\mathrm{s})$ & 343 & 3 & 1 & 1200 & 1200 & 30 \\
& {$[3 ; 1200]$} & {$[0 ; 8]$} & {$[0 ; 3]$} & {$[1200 ; 1200]$} & {$[1200 ; 1200]$} & {$[3 ; 100]$} \\
Gap $(\%)$ & 2 & 0 & 0 & 81 & 33 & 0 \\
& {$[0 ; 29]$} & {$[0 ; 0]$} & {$[0 ; 0]$} & {$[60 ; 91]$} & {$[11 ; 44]$} & {$[0 ; 0]$}
\end{tabular}

Table 2: Results for set A and B instances 


\begin{tabular}{lccccc} 
& set C & set D & set E & set F & set G \\
\hline Variables & 2880 & 8580 & 17280 & 12870 & 25830 \\
Constraints & 1080 & 1981 & 2881 & 2971 & 4321 \\
\#VI & 1084 & 1131 & 1093 & 2534 & 2517 \\
\hline \#Opt & 30 & 29 & 27 & 8 & 3 \\
\hline Gap $_{0}(\%)$ & 5 & 5 & 4 & 5 & 6 \\
& {$[1 ; 13]$} & {$[1 ; 11]$} & {$[2 ; 6]$} & {$[3 ; 10]$} & {$[2 ; 17]$} \\
\#Nodes & 150 & 382 & 440 & 604 & 299 \\
& {$[0 ; 832]$} & {$[74 ; 1385]$} & {$[13 ; 1217]$} & {$[48 ; 1085]$} & {$[30 ; 707]$} \\
CPU $_{\text {IP }}(\mathrm{s})$ & 46 & 248 & 440 & 1089 & 1150 \\
& {$[9 ; 168]$} & {$[60 ; 1200]$} & {$[53 ; 1200]$} & {$[82 ; 1200]$} & {$[144 ; 1200]$} \\
Gap(\%) & 0 & 0.01 & 2 & 3 & 5 \\
& {$[0 ; 0]$} & {$[0 ; 0.13]$} & {$[0 ; 2]$} & {$[0 ; 7]$} & {$[0 ; 15]$}
\end{tabular}

Table 3: Results for set C-G instances obtained with the DLSPSD2* formulation 